\title{
-NOTES-
}

\section{A NOTE ON RIGID BODY DISPLACEMENTS IN THE THEORY OF THIN ELASTIC SHELLS*}

\author{
By P. M. NAGHDI (University of California, Berkeley)
}

1. Introduction. The stress-strain relations appropriate to the linear theory of thin elastic isotropic shells have long been the subject of numerous investigations. Recently, a suitable system of stress-strain relations, which include the effects of both transverse shear deformation and transverse normal stress, has been derived for axisymmetric shells of revolution by E. Reissner [1] and, in general form by the present author [2], where the deformation is referred to the lines of curvature of the middle surface. Also contained in [2] are explicit expressions for the stress resultants $\left\{N_{1}, N_{2}, N_{12}, N_{21}\right\}$, $\left\{V_{1}, V_{2}\right\}$, and the stress couples $\left\{M_{1}, M_{2}, M_{12}, M_{21}\right\}$ in terms of quantities which describe the state of strain, when only the effect of transverse shear deformation is retained (see Eqs. (3.6) of [2]). This latter system of stress-strain relations, in the notation of Ref. [2], is of the form

$$
\begin{aligned}
& N_{12}=G h\left[\left(\gamma_{1}^{0}+\gamma_{2}^{0}\right)+\frac{h^{2}}{12}\left(\frac{1}{R_{1}}-\frac{1}{R_{2}}\right)\left(\frac{\gamma_{1}^{0}}{R_{1}}-\delta_{1}\right)\right], \\
& N_{21}=G h\left[\left(\gamma_{1}^{0}+\gamma_{2}^{0}\right)+\frac{h^{2}}{12}\left(\frac{1}{R_{2}}-\frac{1}{R_{1}}\right)\left(\frac{\gamma_{2}^{0}}{R_{2}}-\delta_{2}\right)\right], \\
& M_{12}=\frac{1-\nu}{2} D\left[\left(\delta_{1}+\delta_{2}\right)+\left(\frac{1}{R_{2}}-\frac{1}{R_{1}}\right) \gamma_{1}^{0}\right], \\
& M_{21}=\frac{1-\nu}{2} D\left[\left(\delta_{1}+\delta_{2}\right)+\left(\frac{1}{R_{1}}-\frac{1}{R_{2}}\right) \gamma_{2}^{0}\right], \text { etc. }
\end{aligned}
$$

which satisfy the identity

$$
N_{12}+\frac{M_{12}}{R_{1}}=N_{21}+\frac{M_{21}}{R_{2}} .
$$

In (1.1), $R_{1}$ and $R_{2}$ are the principal radii of curvature of the middle surface; $h$ is the shell thickness; $D=E h^{3} /\left[12\left(1-\nu^{2}\right)\right] ; G$ is the shear modulus; $E$ and $\nu$ are respectively Young's modulus and Poisson's ratio; and the quantities $\gamma_{1}^{0} \delta_{1}$, etc., which refer to the components of strain, will be defined presently.

The stress-strain relations derived in [2] have been criticized very recently by Goldenveiser [3], who has asserted that for rigid body displacements alone not all quantities on the right-hand side of (1.1) vanish, which implies that the shell space will not remain stress-free. It is the purpose of the present note to examine the implications of rigid body displacements of the shell space, and this may be regarded as an addition to

*Received March 19, 1959; revised manuscript received January 6, 1960. The results presented in this paper were obtained in the course of research sponsored by the Office of Naval Research under Contract Nonr-222(69), Project NR-064-436, with the University of California (Berkeley). 
the results in [2]. Although attention is confined to shells whose stress-strain relations are defined by (1.1), the extension to the case in which the effects of both transverse shear deformation and normal stress are included will be apparent and leads to the same conclusion. It is shown that in the absence of surface loads and body forces, contrary to the assertion made in [3], for rigid body displacements all of the stress resultants and the stress couples vanish; and conversely, the vanishing of these resultants corresponds in fact to the most general form of the rigid body displacements of the shell space.

2. Preliminary background. In order to make the following analysis reasonably self-contained, we recall that the position vector of a generic point in the shell space is defined by

$$
\mathbf{R}\left(\xi_{1}, \xi_{2}, \zeta\right)=\mathbf{r}\left(\xi_{1}, \xi_{2}\right)+\zeta \mathbf{n}
$$

where $\mathbf{r}$ is the position vector of the corresponding point (with coordinates $\xi_{1}$ and $\xi_{2}$ ) on the middle surface, and $-h / 2 \leqq \zeta \leqq h / 2$ is measured along the unit normal $\mathbf{n}$ to the middle surface. Further, we denote by $t_{1}$ and $t_{2}\left(t_{1}, t_{2}\right.$ and $n$ form a right-handed system), the unit tangent vectors to the $\xi_{1}$ - and $\xi_{2}$-parametric curves which will be referred to the lines of curvature on the middle surface $(\zeta=0)$.

The displacement vector, as assumed in [2] and which leads to the results (1.1), is given by

$$
\mathbf{U}\left(\xi_{1}, \xi_{2}, \zeta\right)=\mathbf{u}\left(\xi_{1}, \xi_{2}\right)+\zeta \boldsymbol{\beta}\left(\xi_{1}, \xi_{2}\right),
$$

where

$$
\mathbf{u}=u_{1} \mathbf{t}_{1}+u_{2} \mathbf{t}_{2}+w \mathbf{n}, \quad \beta=\beta_{1} \mathbf{t}_{1}+\beta_{2} \mathbf{t}_{2} .
$$

With the notation $\partial / \partial \xi_{1}(\quad) \equiv(),{ }_{1}$ the quantities $\gamma_{1}^{0}, \delta_{1}$, etc. in (1.1), (or in Eqs. (3.6) of [2]), may be conveniently written as

$$
\begin{aligned}
\epsilon_{1}^{0} & =\frac{\mathbf{u}_{.1} \cdot \mathbf{r}_{.1}}{\alpha_{1}^{2}}, & \epsilon_{2}^{0} & =\frac{\mathbf{u}_{.2} \cdot \mathbf{r}_{.2}}{\alpha_{2}^{2}}, \\
\gamma_{1}^{0} & =\frac{\mathbf{u}_{.1} \cdot \mathbf{r}_{.2}}{\alpha_{1} \alpha_{2}}, & \gamma_{2}^{0} & =\frac{\mathbf{u}_{.2} \cdot \mathbf{r}_{.1}}{\alpha_{2} \alpha_{1}}, \\
\alpha_{1} \gamma_{1 \zeta} & =\boldsymbol{\beta} \cdot \mathbf{r}_{.1}+\mathbf{u}_{, 1} \cdot \mathbf{n}, & \alpha_{2} \gamma_{25}^{0} & =\boldsymbol{\beta} \cdot \mathbf{r}_{.2}+\mathbf{u}_{, 2} \cdot \mathbf{n}, \\
\kappa_{1} & =\frac{\boldsymbol{\beta}_{.1} \cdot \mathbf{r}_{\cdot 1}}{\alpha_{1}^{2}}, & \kappa_{2} & =\frac{\boldsymbol{\beta}_{.2} \cdot \mathbf{r}_{.2}}{\alpha_{2}^{2}}, \\
\delta_{1} & =\frac{\boldsymbol{\beta}_{.1} \cdot \mathbf{r}_{.2}}{\alpha_{1} \alpha_{2}}, & \delta_{2} & =\frac{\boldsymbol{\beta}_{.2} \cdot \mathbf{r}_{\cdot 1}}{\alpha_{2} \alpha_{1}},
\end{aligned}
$$

where $\left(\alpha_{1}^{2}, \alpha_{2}^{2}\right)$ are the non-vanishing components of the middle surface metric.

3. Rigid body displacements of the shell space. Let, in the absence of surface loads and body forces, the displacements be specified by

$$
\mathbf{U}=\mathfrak{u}_{0}+\omega \times \mathbf{R},
$$

which, by (2.1), may also be written as

$$
\mathbf{U}=\left(\mathbf{u}_{0}+\boldsymbol{\omega} \times \mathbf{r}\right)+\zeta(\boldsymbol{\omega} \times \mathbf{n}),
$$

where $\mathbf{u}_{0}$ and the rotation $\omega$ are constant vectors. The displacement vector defined by (3.1) represents the most general form of rigid body displacements of the shell space, 
and includes as special cases purely rigid body translation and purely rigid body rotation.

Returning now to our main objective, we substitute (3.1) into the first six of (2.4) to obtain

$$
\begin{aligned}
\epsilon_{1}^{0} & =\frac{1}{\alpha_{1}^{2}} \mathbf{r}_{, 1} \cdot\left(\omega \times \mathbf{r}_{, 1}\right)=0, \\
\gamma_{1}^{0} & =\frac{1}{\alpha_{1} \alpha_{2}} \mathbf{r}_{, 2} \cdot\left(\omega \times \mathbf{r}_{, 1}\right)=\omega \cdot \mathbf{n}, \\
\gamma_{15}^{0} & =\frac{1}{\alpha_{1}}\left[\mathbf{r}_{, 1} \cdot \omega \times \mathbf{n}+\mathbf{n} \cdot \omega \times \mathbf{r}_{, 1}\right], \\
& =\frac{1}{\alpha_{1}}\left[\mathbf{r}_{, 1} \cdot \omega \times \mathbf{n}-\mathbf{r}_{, 1} \cdot \omega \times \mathbf{n}\right]=0,
\end{aligned}
$$

and similarly,

$$
\epsilon_{2}^{0}=0, \quad \gamma_{2}^{0}=-\omega \cdot \mathbf{n}, \quad \gamma_{2 \zeta}^{0}=0 .
$$

Likewise, substitution of (3.1) into the remaining expressions of (2.4), with the aid of $\mathrm{n}_{, 1}=\mathbf{r}_{1,} / R_{1}$ and $\mathbf{n}, 2_{2}=\mathbf{r}, 2 / R_{2}$, yields

$$
\begin{aligned}
\kappa_{1} & =\frac{1}{\alpha_{1}^{2}} \omega \times \mathbf{n}_{, 1} \cdot \mathbf{r}_{, 1}=\frac{1}{\alpha_{1}^{2}} \omega \cdot \mathbf{n}_{, 1} \times \mathbf{r}_{, 1}=0, \\
\delta_{1} & =\frac{1}{\alpha_{1} \alpha_{2}} \omega \times \mathbf{n}_{, 1} \cdot \mathbf{r}_{, 2}=\frac{1}{\alpha_{1} \alpha_{2}} \omega \cdot \frac{\mathbf{r}_{.1} \cdot \mathbf{r}_{2}}{R_{1}}, \\
& =\frac{\omega \cdot \mathbf{n}}{R_{1}}
\end{aligned}
$$

and similarly,

$$
\kappa_{2}=0, \quad \delta_{2}=-\frac{\omega \cdot \mathbf{n}}{R_{2}} .
$$

Next, substituting (3.2) and (3.3) into (1.1) and observing that

$$
\begin{gathered}
\gamma_{1}^{0}+\gamma_{2}^{0}=\frac{\gamma_{1}^{0}}{R_{1}}-\delta_{1}=\frac{\gamma_{2}^{0}}{R_{2}}-\delta_{2}=0 \\
\left(\delta_{1}+\delta_{2}\right)+\left(\frac{1}{R_{2}}-\frac{1}{R_{1}}\right) \gamma_{1}^{0}=\left(\delta_{1}+\delta_{2}\right)+\left(\frac{1}{R_{1}}-\frac{1}{R_{2}}\right) \gamma_{2}^{0}=0,
\end{gathered}
$$

it follows that in the absence of surface loads and body forces, all of the resultants in (1.1) vanish. Furthermore, it may be easily verified that when in the absence of surface loads and body forces all resultants are set equal to zero, the solution of differential equations (1.1) and (2.3), with an appeal to the uniqueness theorem, is indeed given by (3.1).

Note added in proof: Professor Goldenweiser has recently informed the author that he is in agreement with the content of this note.

\section{REFERENCES}

1. Eric Reissner, Stress strain relations in the theory of thin elastic shells, J. Math. Phys. 29, 90-95 (1950)

2. P. M. Naghdi, On the theory of thin elastic shells, Quart. Appl. Math. 14, 369-380 (1957)

3. A. L. Goldenveiser, Rev. No. 4425 of Ref. [2], (in Russian), Referat. Zhur., No. 4, 1958 